\title{
Ascorbic acid and risk of kidney stones
}

Men who take ascorbic acid supplements (a form of vitamin C) are twice as likely to develop kidney stones as men who do not take any dietary supplements, say the authors of a research letter published in JAMA Internal Medicine. "High doses of vitamin $\mathrm{C}$ have long been suspected to increase the risk of kidney stones since some vitamin $\mathrm{C}$ is excreted as oxalate-one of the key components of kidney stones," explains researcher Agneta Åkesson. "However, there has been considerable uncertainty as to whether vitamin $\mathrm{C}$ supplements are associated with kidney stone risk."

To investigate the association between vitamin $\mathrm{C}$ supplements and the formation of kidney stones, Åkesson and colleagues followed-up 23,355 men from a large population-based cohort who had no history of kidney stones and who took either no dietary supplements or ascorbic acid supplements only (approximately $1,000 \mathrm{mg}$ ). For comparison purposes, the researchers repeated the analysis for multivitamin users. They used health registry data to identify first incident cases of kidney stones.

Over a mean 11 years of follow-up, 436 participants developed kidney stones. Ascorbic acid supplement use was associated with an approximately twofold increased risk of kidney stones. "The risk of kidney stones among men taking vitamin $\mathrm{C}$ supplements was highest amongst those taking them at least once a day," adds Åkesson. By contrast, multivitamin use was not associated with kidney stone risk.

The researchers say that their findings need to be confirmed by other studies, but suggest that high-dose preparations of vitamin $\mathrm{C}$ should be avoided, particularly by individuals with a history of kidney stones.

\section{Susan J. Allison}

Original article Thomas, L. D. et al. Ascorbic acid supplements and kidney stone incidence among men: a prospective study. JAMA Intern. Med. doi:10.1001/ jamainternmed.2013.2296 